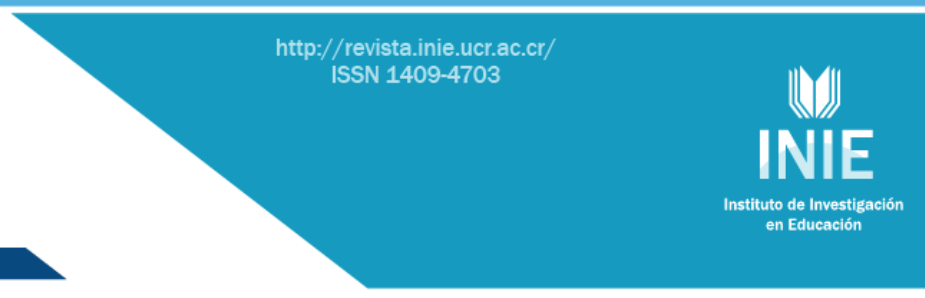

\title{
INTRODUCCIÓN DE TECNOLOGÍAS EN EL AULA DE DOS PREESCOLARES PÚBLICOS COSTARRICENSES: ESTRATEGIAS DE AUTOGESTIÓN, ALCANCES Y LIMITACIONES.
}

AN INTRODUCTION OF TECHNOLOGY IN THE CLASSROOM OF TWO COSTA RICAN PUBLIC PRESCHOOLS: SELF-MANAGEMENT STRATEGIES, POSSIBILITIES AND LIMITATIONS

\section{Volumen 13, Número 2} Mayo - Agosto

pp. $1-23$

Este número se publicó el 30 de mayo de 2013

\author{
María de los Ángeles Calderón Jiménez \\ Michael Padilla Mora \\ Jaime Fornaguera Trías
}

Revista indizada en REDALYC, $\underline{\text { SCIELO }}$

Revista distribuida en las bases de datos:

CATÁLOGO DE LATINDEX, IRESIE, CLASE, DIALNET, DOAJ, E-REVIST@S, SHERPA/ROMEO, QUALIS, MIAR

Revista registrada en los directorios:

ULRICH'S, REDIE, RINACE, OEI, MAESTROTECA, PREAL, CLASCO 


\title{
INTRODUCCIÓN DE TECNOLOGÍAS EN EL AULA DE DOS PREESCOLARES PÚBLICOS COSTARRICENSES: ESTRATEGIAS DE AUTOGESTIÓN, ALCANCES Y LIMITACIONES. \\ AN INTRODUCTION OF TECHNOLOGY IN THE CLASSROOM OF TWO COSTA RICAN PUBLIC PRESCHOOLS: SELF-MANAGEMENT STRATEGIES, POSSIBILITIES AND LIMITATIONS
}

\author{
María de los Ángeles Calderón Jiménez ${ }^{1}$ \\ Michael Padilla Mora ${ }^{2}$ \\ Jaime Fornaguera Trías ${ }^{3}$
}

\begin{abstract}
Resumen: Este artículo sistematiza el proceso de introducción de la tecnología dentro de dos aulas de Preescolar, específicamente el uso de la computadora como un área más dentro del periodo "Juego-Trabajo", en dos jardines de niños y niñas de la comunidad de Tres Ríos, Cartago. Se detalla el origen de las propuestas y el proceso desarrollado mediante la autogestión del recurso, ya que el sistema educativo costarricense no cuenta con una política generalizada en el uso de la tecnología accesible al nivel de Preescolar. La información se adquirió por medio de entrevistas a directoras y cuestionarios a docentes; además, se observó la conducta de los niños al enfrentarse a la tecnología en el aula y se documentaron los alcances y las limitaciones percibidas por docentes y administrativas, así como sugerencias de los involucrados para que esfuerzos de este tipo sean viables y sostenibles en el tiempo. Se concluye que cuando hay una visión y propósitos comunes, a partir de un liderazgo que coordine la acción, el diseño y la ejecución de un plan estratégico escolar que se deriva de las necesidades que hay que resolver, es posible lograr proyectos, a pesar de no contar con el apoyo directo de instancias políticas y gubernamentales.
\end{abstract}

Palabras clave: TECNOLOGÍAS, PREESCOLAR, AUTOGESTIÓN, PERIODO JUEGO-TRABAJO, COSTA RICA

\begin{abstract}
This article systematizes the introduction process of technology inside two preschool classrooms, specifically, the use of a computer as an additional area inside the play and work time, in two children gardens community of Tres Ríos, Cartago. It details the proposals' origin and the developed process from the resource's self-management, given that the Costa Rican educational system lacks a general policy in the use of accessible technologies at the preschool level. All the data was gathered from interviews to principals and written forms to teachers, and also from observation to students' behavior when it came to using the technology inside their classroom. The possibilities and limitations noticed by teachers and staff, in addition to suggestions from people involved so this kind of projects can be possible and sustained over time were documented. Concluding, when there's a vision and will in common, starting from a leadership that coordinates the actions, design and development of a strategic plan that comes from the needs that must be resolved, it's possible to achieve projects, even when there's no direct support from political instances.
\end{abstract}

Key words: TECHNOLOGIES, PRESCHOOL, SELF-MANAGEMENT, PLAYWORK PERIOD, COSTA RICA

\footnotetext{
${ }_{1}^{1}$ Docente de Educación Preescolar en el Ministerio de Educación Pública. Asistente en el Programa de Investigación en Neurociencias de la Universidad de Costa Rica. Licenciada en Educación Preescolar de la universidad de Costa Rica. Bachiller en Psicología de la Universidad de Costa Rica. Dirección electrónica: maricj73@gmail.com.

${ }^{2}$ Investigador del Centro de Investigación en Neurociencias y Profesor de la Escuela de Psicología de la Universidad de Costa Rica (UCR). Máster en Ciencias Cognoscitivas de la Universidad de Costa Rica. Bachiller en Filosofía con énfasis en Ciencias Sociales de la Universidad Nacional. Dirección electrónica: michaelpadillamora@gmail.com

${ }^{3}$ Profesor del Departamento de Bioquímica de la Escuela de Medicina de la Universidad de Costa Rica y Director del Centro de Investigación en Neurociencias de la Universidad de Costa Rica. Bachiller en Biología, Máster en Fisiología, Universidad de Costa Rica y Dr. Rer.Nat. de la Universidad de Düsseldorf, Alemania. Dirección electrónica: jfornagu@gmail.com
}

Artículo recibido: $1^{\circ}$ de octubre, 2012

Aprobado: 20 de mayo, 2013

Volumen 13, Número 2, Año 2013, ISSN 1409-4703 


\section{INTRODUCCIÓN}

Las Tecnologías de la Información y de la Comunicación (TIC) hacen posible la transmisión de datos, textos e imágenes en forma digital y en tiempo real, y permiten a personas, organizaciones e instituciones públicas automatizar sus procesos de búsqueda e intercambio de información. Además, promueven la creación de redes de interacción, que involucran la radio, los teléfonos móviles, las computadoras y distintos programas de cómputo en el contexto de redes globales como la Internet (Monge y Hewwitt, 2004). Por estas y otras características, su uso se ha convertido en algo cotidiano, popular y necesario para alcanzar un mayor aprovechamiento del tiempo y de otros recursos en el contexto de nuestras sociedades.

Actualmente, las TIC han modificado los escenarios cotidianos de niños, niñas, jóvenes y adultos. Es común observar su uso generalizado en centros de salud, servicios de atención al cliente y centros de enseñanza, e incluso en el hogar; en ese sentido, las computadoras y los dispositivos móviles se han convertido en recursos frecuentes para niños y niñas que observan a sus padres y madres utilizar este tipo de equipo durante sus actividades de trabajo y ocio. Poco a poco, las nuevas generaciones, 'nativas digitales' (Cabra y Marciales, 2009), van creciendo y aprendiendo a utilizar la gran variedad de TIC por sí mismos y para diferentes propósitos.

Ante este contexto, es relevante que los centros de enseñanza del sistema de educación pública propicien la introducción de las TIC como apoyo y recurso pedagógico, tanto para docentes como para estudiantes, donde a estos últimos se les permita accesar a la tecnología, colaborando así en la ampliación de sus posibilidades de inserción social en el futuro al tener una mejor formación tanto académica como tecnológica (Bennett, Maton y Kervin, 2008). Esto vendría a colaborar con la reducción de la brecha de desigualdad ante las posibilidades que ofrecen los sistemas privados (Mesa, Sánchez y Caro 2008), por cuanto estos, al no tener limitaciones de tipo económico, cuentan con más herramientas tecnológicas para el uso en el aula. De otra manera, según Camacho (2009), podría en cambio perpetuarse la marginalidad de algunos grupos que, aislados del conocimiento y la utilización de muchos instrumentos tecnológicos, pierden importantes posibilidades de introducirse en actividades sociales vinculadas con el intercambio de comunicación e información. 
En la actualidad, las TIC se han vuelto esenciales para el desarrollo económico, político y social de los países, donde se busca que todas las personas tengan acceso al conocimiento. La ausencia de una política de Tecnologías de la Información y de la Comunicación en instituciones públicas, puede aumentar la desigualdad entre los países y las personas (México, Secretaría de Educación Pública, 2011). Sistemas educativos públicos de diversos países comparten la característica de no contar con suficientes recursos para suplir las necesidades de sus instituciones, dando lugar a la autogestión como una forma de organización dirigida a la consecución de proyectos considerados vitales para mejorar la calidad de la educación que reciben sus estudiantes.

La autogestión, a su vez, favorece la innovación al colaborar para que instituciones educativas de bajos recursos económicos adquieran tecnología, tratando de mejorar la calidad en la educación al proveer de nuevas oportunidades a los estudiantes, tal es el caso de México, donde a través de esta gestión, la secretaria de Educación Pública, busca recuperar el papel relevante de la escuela para dar respuesta a una sociedad que demanda ciudadanos competentes, que enfrenten y superen los desafíos; una escuela que se posicione como el espacio idóneo para la ampliación de oportunidades de aprendizaje, donde estén involucrados todos los actores escolares: directores, docentes, alumnos, padres y madres de familia y miembros de la comunidad.

Para que la autogestión en los centros educativos pueda ser realizada, debe establecerse una visión y propósitos comunes para todos los involucrados, a partir de un liderazgo que coordine la acción, el diseño y la ejecución de un plan estratégico escolar que se derive de las necesidades que hay que resolver, con evaluación permanente de los logros alcanzados y de las nuevas necesidades que vayan surgiendo. El proceso implica una comunicación fluida entre todas las partes, relaciones personales horizontales, diseño de objetivos, estrategias y metas compartidas, así como el compromiso para su ejecución y logro, además de la retroalimentación para evaluar el proceso y mejorarlo de ser necesario (Parra y Wondon, 2011).

De esta forma, la gestión en el Centro Educativo da un viraje positivo, donde las relaciones de colaboración son esenciales, dejando atrás el aislamiento profesional, las visiones divergentes, la escasa comunicación, la ausencia de liderazgo directivo y la limitada participación de los padres de familia, además de la desarticulación de iniciativas y acciones. De este modo, la institución promoverá condiciones para que sea atractiva para los alumnos 
y apreciada por la comunidad, donde todos colaboren en alcanzar los objetivos propuestos (México, Secretaría de Educación Pública, 2011).

En el caso específico de la Educación Preescolar, y partiendo de esta como la etapa que cimienta las bases del desarrollo del individuo de forma integral, involucrando las características del medio físico, natural y social, la introducción de tecnologías, especialmente computacionales, es clave para ampliar y enriquecer nuevos conocimientos en los estudiantes, que los capaciten para enfrentar situaciones novedosas fuera del aula (Haugland y Wright, 2000).

Desde esta perspectiva, y en correspondencia con la idea de un currículo que ofrece a niños y niñas un ambiente integrado a través de su aula (Costa Rica, Ministerio de Educación Pública, 2004), el uso de la computadora dentro del salón de clase puede servir como apoyo lúdico-didáctico, capaz de complementar las actividades cotidianas por medio de la construcción de nuevos conocimientos. Dado lo anterior, y partiendo de una Educación Preescolar basada en guías que orienten el aprendizaje y la interacción a través del juego (Plowman, Stephen y McPake, 2010), es posible que la inclusión y la colocación estratégica de materiales digitales en el ambiente pedagógico cotidiano y al alcance de niños y niñas logre favorecer su uso espontáneo y autónomo en actividades generadoras de conocimiento (Camacho y González, 2008).

La incorporación de las TIC al contexto específico de la Educación Preescolar ha sido controversial y muchas veces ignorada. El alto costo de los equipos y su uso para el aprendizaje de conceptos básicos, el tiempo que invierten los niños en el uso del computador vs las actividades que pueden promover el desarrollo de destrezas comunicativas, así como la elevada venta de software comercial, generan controversias en cuanto al uso entre las personas ligadas al mundo de la Educación Preescolar (Garassini y Padrón, 2004). Algunos han optado por descartar la introducción de la computadora en los centros educativos de Educación Preescolar, pero otros, mediante la estrategia de autogestión de recursos, procuran incluirla como un área más en el aula. Un espacio de libre elección por parte de niños y niñas, donde el periodo de exposición es de aproximadamente 45 minutos semanales.

Actualmente, en el sistema nacional de Educación Preescolar costarricense no existe una estrategia oficial generalizada para el uso de equipo computacional en los ciclos de 
materno y transición, pero sí destacan diferentes iniciativas orientadas hacia estos propósitos, que se pueden organizar en 3 líneas de trabajo:

PRONIE: el Ministerio de Educación Pública (MEP) ha realizado importantes esfuerzos para incorporar la informática a la Enseñanza General Básica a través del Programa Nacional de Informativa Educativa (PRONIE), con la colaboración de la Fundación Omar Dengo (FOD). En la actualidad, existe cobertura de programas informáticos en los ciclos I y II de la educación pública nacional, y aunque aún no se ha establecido una política de cobertura adaptada a las necesidades del currículo de Educación Preescolar se está trabajando en ello. Mientras tanto, PRONIE propicia que algunos Jardines de Niños tengan acceso al Laboratorio de Cómputo de sus escuelas anexas, donde desarrollan una estrategia de aprendizaje basada en proyectos, orientada hacia la implementación de los estándares de desempeño definidos para este nivel educativo (Zúñiga y Brenes, 2008). Asimismo, en el caso de algunas instituciones multigrado, conocidas como unidocentes, los estudiantes de diferentes niveles comparten un mismo espacio (Angulo, Cerdas y Ovares, 2010), donde PRONIE favorece que los Preescolares tengan contacto con equipos de cómputo instalados en los salones generales.

Ambientes Virtuales Colaborativos: la Dirección Curricular del MEP, el Departamento de Educación Preescolar (DEP), el Centro de Investigación en Neurociencias (CIN) de la Universidad de Costa Rica y el Laboratorio de Investigación en Ingeniería Biomédica (LIIB) de la Escuela de Ingeniería Eléctrica, también de la Universidad de Costa Rica, han promovido la incorporación de la tecnología dentro del salón de Preescolar. Esta iniciativa ha favorecido que el equipo de cómputo pueda ser introducido como otra área más, como la de construcción, la de arte o la de ciencias, por citar algunas, en correspondencia con los programas de estudio vigentes para los Ciclos de Materno Infantil y de Transición (Costa Rica, MEP, 2000). La iniciativa destaca por la utilización exclusiva de software libre. Se trabaja con el sistema operativo Edubuntu y sus aplicaciones educativas y otras, utilizando siempre software libre como el programa para la creación de multimedia interactiva Blender (http://www.blender.org/). 
En los Ambientes Virtuales Colaborativos, los niños y niñas se enfrentan a juegos virtuales en los que deben resolver problemas de manera conjunta. La meta u objetivo del juego solo puede alcanzarse si los niños trabajan juntos, utilizando diferentes interfases (ratón, teclado, etc.). De esta manera, se propicia la socialización, el desarrollo cognitivo y la alfabetización tecnológica, entre otros (MEP y Fundación Omar Dengo, 2011).

Iniciativas institucionales autogestionadas: en busca de soluciones, ante la clara necesidad y con gran iniciativa, algunas instituciones Preescolares han autogestionado notables proyectos para incorporar la tecnología a sus aulas. Con el apoyo del personal docente y administrativo, padres y madres de familia, así como con el soporte de diversos actores de la comunidad en general, varias instituciones se han organizado, estableciendo estrategias de autofinanciamiento (a partir de rifas, ventas, colaboración de las familias, etc.) para comprar equipo de cómputo. Todo esto con el fin de responder a las demandas de la era digital donde se desarrollan los Preescolares, ofreciéndoles, así, mejores oportunidades. Estas iniciativas incorporan, en una etapa posterior, la computadora dentro de un espacio adicional del salón de clases, proporcionándole a los niños una opción novedosa para realizar otras actividades dentro de su rutina diaria (Camacho y González, 2008). De esta manera, se apoya y fortalece el aprendizaje y, además, se facilita la labor de la docente.

Por todo lo anterior, este artículo se centra en las iniciativas institucionales autogestionadas y ofrece una sistematización de la experiencia de dos Centros Educativos Preescolares Públicos de Costa Rica, que han llevado adelante estos proyectos. Específicamente, se abordan los casos del Jardín de Niños José Ana Marín, ubicado en Vásquez de Coronado (provincia de San José) y el Jardín de Niños Central de Tres Ríos (provincia de Cartago). Estos jardines han logrado introducir las TIC en la cotidianeidad de las aulas, gracias a un esfuerzo significativo desde muchas partes, con el objetivo de ofrecer mejores oportunidades para las comunidades de niños y niñas que conforman cada institución.

La investigación presenta un resumen del proceso partiendo desde la identificación de la necesidad, hasta la obtención del equipo, mediante la estrategia de autogestión, 
enfatizando, además, uno de los retos más importantes de estas iniciativas: el establecimiento de la metodología del uso en el aula. También, se destacan algunos de los aciertos, alternativas y dificultades que han enfrentado las instituciones durante la ejecución de los proyectos, para dar lugar a un análisis de sus eventuales alcances y limitaciones y de cómo solucionarlas.

\section{METODOLOGÍA}

El enfoque exploratorio de la investigación que se implementó fue la recolección de datos cualitativos, involucrando diversas estrategias como la entrevista, los cuestionarios y la observación. El contacto inicial con las dos instituciones educativas se realizó por medio del Departamento de Educación Preescolar (DEP) del Ministerio de Educación Pública (MEP), en el contexto de sus proyectos conjuntos con el Centro de Investigación en Neurociencias de la Universidad de Costa Rica.

Identificación y contacto con Instituciones: se coordinó con el DEP para obtener información sobre instituciones públicas de Educación Preescolar que contaran con tecnologías computacionales como recurso dentro de todas sus aulas (incluyendo los ciclos de materno y transición). EI DEP facilitó una base de datos con instituciones que cumplían con los criterios para la investigación y de ellas se escogieron dos (Costa Rica, Departamento de Estadística, MEP, 2010). Luego, vía telefónica, se estableció contacto con las directoras de estas instituciones para determinar su anuencia a colaborar con el desarrollo de la investigación.

Participantes: gracias al contacto previamente establecido con el DEP y a la buena disposición del personal docente y administrativo de las instituciones identificadas, fue posible entrevistar a 10 docentes de cada jardín Preescolar. En específico, 6 docentes de ciclo transición y 4 de ciclo materno del Jardín Central de Tres Ríos y 7 docentes de ciclo transición y 3 del ciclo de materno en el Jardín de Niños y Niñas José Ana Marín (Coronado), todas las docentes llenaron además un cuestionario y las directoras de ambos centros educativos fueron entrevistadas. La mayoría de las docentes del Jardín de Niños y Niñas José Ana Marín y del Jardín Central de Tres Ríos son licenciadas en Educación de Preescolar y con un promedio de 19 y 18 años de servicio. 
Entrevistas: se gestionaron dos citas en cada Centro Educativo para obtener entrevistas con las directoras. Utilizando una guía semi-estructurada, en la primera cita se indagó sobre el proceso de introducción y auto-gestión de tecnologías computacionales en cada institución, ¿de dónde surgió la idea?, ¿cómo se realizó el proyecto?, ¿cuántas máquinas lograron obtener?, ¿cuántos niños fueron y están siendo beneficiados?, apoyo recibido, financiamiento del equipo a través del tiempo, capacitación obtenida, logros y limitantes del proyecto, actitud de docentes y niños ante la incorporación de la tecnología dentro del aula y software con que trabajan. La segunda entrevista se hizo con el objetivo de precisar aspectos que no habían quedado claros en la primera, especialmente respecto al proceso de adquisición y uso del equipo. Cada entrevista tuvo una duración promedio de 60 minutos. Algunas de las preguntas utilizadas fueron: “¿Cómo surgió el interés de introducir el uso de la computadora en el aula de Preescolar?" o "¿Qué logros y limitaciones han encontrado al introducir las computadoras en las aulas?"

Cuestionarios: previa autorización y coordinación con las directoras, se distribuyeron cuestionarios con preguntas abiertas para las docentes en dos etapas. En la primera, los cuestionarios estaban dirigidos a recabar información general sobre ellas: nivel académico, años de servicio en educación, años de trabajar en la institución. Además, se les preguntó sobre si conocían sobre los orígenes del proyecto, capacitaciones obtenidas, metodología utilizada para el uso de las tecnologías computacionales en la cotidianidad del aula, software utilizado, logros y limitaciones percibidas como parte de estos procesos. En una segunda etapa se indagó sobre aspectos específicos como el nombre que le daban a esa área o espacio, los objetivos de trabajo, el primer acercamiento de los niños y niñas a la computadora y sobre aspectos de infraestructura física que deben tomarse en cuenta para poder tener la máquina en el aula. Algunos ejemplos de las preguntas incluidas en estos cuestionarios son: “¿En qué momentos de la rutina diaria los niños y niñas tienen contacto con la computadora?” y “¿Qué características físicas consideran para tener el área o espacio donde está la computadora?".

Observación en el Aula: con el propósito de verificar parte de la información suministrada por las directoras y las maestras acerca de las metodologías implementadas y el tipo de recursos multimedia ofrecidos en el trabajo diario, se realizó una observación de la dinámica del aula 
en cada institución, durante aproximadamente 45 minutos, periodo en el cual los estudiantes trabajaron con la computadora. Ahí se observó la metodología implementada, la actitud de los niños, niñas y docentes frente a la computadora, desenvolvimiento de los involucrados, juegos utilizados y reacción de los otros niños y niñas que no usaron el equipo en ese día específico.

Sistematización y síntesis: una vez recolectada la información se sistematizó y sintetizó mediante los distintos instrumentos, de acuerdo con los siguientes ejes:

Recapitulación del proceso de auto-gestión: reseña sobre la forma en que cada una de las dos instituciones logró obtener su equipo. Se contemplaron los eventos que motivaron el inicio del proyecto y las estrategias implementadas hasta llegar a adquirir las computadoras para cada una de las aulas. Esta información se obtuvo mediante las entrevistas a las directoras, así como a partir de otros aportes de las docentes y quienes reafirmaron lo comentado por las primeras.

Metodologías implementadas y Software: se describe la forma mediante la cual las docentes emprenden el uso de la computadora con sus alumnos en el aula. Se detalla la organización implicada, generalidades del software usado, así como la manera y los momentos de la jornada diaria cuando la tecnología es aprovechada. Los cuestionarios aplicados a docentes y la observación directa en el aula fueron los insumos para esta sección.

Alcances y limitaciones del proyecto: se explicitan los logros alcanzados por las iniciativas según el criterio de docentes y directoras. Además, se sintetizan los obstáculos o dificultades que han tenido lugar. Estos datos se obtuvieron a través de las entrevistas a las directoras y los cuestionarios a las docentes.

A continuación, se expone la sistematización en términos de los ejes descritos para el caso de cada una de las instituciones abordadas. 


\section{RESULTADOS}

\subsection{Sistematización}

\subsubsection{Recapitulación del proceso de auto-gestión:}

\subsubsection{La experiencia del Jardín de Niños José Ana Marín}

El Jardín de Niños José Ana Marín, ubicado en Vásquez de Coronado (cantón 11 de la provincia de San José), emprendió su iniciativa de autogestión en el año 2003. En aquel momento, la institución contaba únicamente con una máquina de escribir para labores administrativas.

Ante este panorama, la entonces directora, M.Sc. Lorena Salas Soto, inició los trámites con la Junta de Educación para adquirir una computadora con miras a utilizarla en labores administrativas. El Jardín adquirió el equipo y rápidamente se comenzó a evidenciar su utilidad, no solo como apoyo para la dirección, sino también para labores docentes como procesador de textos. Fuera de horario, las docentes solicitaban el uso del equipo para hacer circulares, trabajos de comités, cartas de referencia de estudiantes y expedientes, además del planeamiento de aula. Este tipo de demanda aumentó paulatinamente hasta que se planteó la necesidad de adquirir otra computadora para uso exclusivo de las docentes, que se adquirió un año después por medio de la Junta de Educación y se colocó en una sala fuera de la oficina de la dirección. En poco tiempo, el incremento en su uso evidenció que dos computadoras no eran suficientes para atender las necesidades de todo el personal docente.

En al año 2004, a través de la gestión de la M.Sc. María Isabel Molina Fallas, docente de la institución y con el visto bueno de la directora, se obtuvo otra computadora. Esto gracias a una donación por parte de la empresa CEFA (distribuidora de productos farmacéuticos y de cuidado personal). Esta empresa renovó su equipo de cómputo en ese año, ofreciendo el equipo usado a varias instituciones que pudieran requerirlo. Molina envió una carta al departamento de informática de CEFA, la empresa accedió a la solicitud y esta docente colocó el equipo en su aula de Preescolar, buscando favorecer que sus alumnos y alumnas tuvieran contacto con este tipo de herramienta tecnológica. Para la docente, se trató de una oportunidad para ofrecer a niños y niñas de escasos recursos, su primer contacto con la computadora. El software lo adquirió con sus propios recursos y consistió, específicamente, en juegos educativos que respondían a la edad de sus estudiantes y cuyo contenido estaba acorde al plan de estudios. La experiencia dio excelentes resultados como 
complemento a las actividades de aula. Los padres y madres de familia se sorprendieron de forma positiva al enterarse de esta nueva opción de aprendizaje ofrecida para sus hijos e hijas, esto según lo consignado en la entrevista correspondiente.

Lo anterior motivó a otras maestras, quienes solicitaron el apoyo entre padres y madres de familia. A este llamado respondió una familia, que hizo algunos trámites y logró que otra empresa donara a la institución un equipo usado. De este modo, se consiguió que en dos aulas se ofrecieran tecnologías computacionales como apoyo a la actividad cotidiana. No obstante, el equipo usado supuso más necesidades de apoyo informático y mantenimiento, lo cual se convirtió en un elemento central, debido a que las computadoras presentaron diferentes desperfectos en software y hardware que dificultaban su uso. Lo anterior, unido a una sobredemanda en las computadoras empleadas para labor docente, promovió que se presentara a la dirección una propuesta para adquirir equipo nuevo. La directora de la institución apoyó la iniciativa y promovió la dotación de equipo nuevo de cómputo a cada una de las 6 aulas. El personal docente y administrativo del Jardín de Niños se comprometió a involucrar a la comunidad en general, a los padres y madres de familia, para que participaran del proyecto en beneficio de los estudiantes del Jardín de Niños.

Es así como en el año 2008 inició el proyecto institucional para dotar a cada aula de su computadora. Se motivó a los padres y madres de familia para que colaboraran económicamente para invertir en equipo. Además, para este fin se utilizó lo recaudado por concepto de la cuota voluntaria de matrícula. En una reunión de personal y en forma conjunta, se decidió que el nuevo equipo adquirido sería para uso exclusivo de los estudiantes en el periodo de "Juego-Trabajo", de la docente como herramienta de apoyo para el aprendizaje y para uso en labores administrativas fuera del horario lectivo. Con el dinero recaudado se compraron los 6 CPU para cada una de las aulas, teclados y ratones (o "mouse"); sin embargo, no fue suficiente para adquirir los monitores.

La directora y las docentes decidieron que el monitor de cada aula se adquiriría, ya sea con cuotas que aportaran las familias o con donaciones según la organización interna de cada grupo. Este procedimiento resultó exitoso, lográndose que todas las aulas contaran con el equipo completo y nuevo. De esta forma, se vieron beneficiados aproximadamente 280 niños y niñas de los ciclos de materno infantil y transición distribuidos en diez grupos. El equipo comenzó a ser utilizado en los primeros meses del año 2008 y a partir de ese momento cada una de las docentes fue responsable del que tenía en su aula. Algunos 
familiares de los niños y niñas, que poseían conocimientos técnicos, colaboraron con el mantenimiento del equipo; pero también fue necesaria la contratación de un técnico, quien se financió por medio del patronato escolar. Es así como hasta el día de hoy, 10 años después que empezara la iniciativa, el proyecto se ha desarrollado y mantenido con sus propios medios, gracias al apoyo de padres, madres, patronato escolar y comunidad educativa en general. En el año 2012, la población beneficiada fue de 315 niños y niñas, pertenecientes a 14 grupos, que usaban 20 computadoras, 6 que compró la institución con la iniciativa y las 14 restantes fueron una donación del MEP, producto del trabajo conjunto con el Centro de Investigación en Neurociencias de la Universidad de Costa Rica y la empresa privada.

\subsubsection{El caso del Jardín de Niños Central de Tres Ríos}

El Jardín Central de Tres Ríos, ubicado en el distrito primero del Cantón La Unión (provincia de Cartago), es actualmente administrado por la Licda. Flory Sanabria López. Mediante una beca otorgada por las Embajadas de Israel y España, con el apoyo del Ministerio de Educación Pública (MEP), la Licda. Sanabria tuvo la oportunidad de visitar Israel en el año 1999 y España en el 2000. En estos viajes se inspiró para impulsar algunas innovaciones que consideró apropiadas para la institución que tiene a cargo. En ambos países observó que el salón de clases contaba con una computadora y dos sillas, lo que propiciaba la interacción y el trabajo en parejas. El uso del equipo por parte de los estudiantes era monitoreado por la docente durante la semana, a fin de que todos los niños tuvieran la misma oportunidad de usarla. Los niños trabajaban con programas educativos que les proveía la docente, correspondientes a su edad, en los cuales se reforzaban conceptos que se estudiaban en clase, funcionan como un complemento y no como un sustituto.

Ya en Costa Rica y como parte del compromiso adquirido al obtener la beca, la Licda. Sanabria compartió la experiencia vivida con docentes de su región y poco a poco emprendió la adaptación de algunas ideas innovadoras en su institución. En el año 2003 decidió plantear la introducción de equipo de cómputo en todas las aulas como un proyecto institucional, esperando a que el nuevo espacio funcionara como un área más en el periodo de "Juego-Trabajo", periodo destinado a que los niños, de forma voluntaria, decidían en qué iban a trabajar. 
Dado que el Patronato Escolar es un ente autorizado para organizar actividades de recaudación de fondos orientadas al desarrollo de proyectos que beneficien a los estudiantes (Poder Ejecutivo de Costa Rica, 2003), se coordinó con sus miembros a fin de realizar actividades para obtener el dinero necesario. También, fue importante la colaboración de las docentes, padres, madres y comunidad educativa en general. En reunión con las familias se explicaron los alcances del proyecto, haciendo hincapié en su importancia e instando a la colaboración en las actividades que se organizaran. En este contexto, un padre de familia y estudiante de Ingeniería en Sistemas ofreció realizar su trabajo Comunal Universitario (TCU) llevando a cabo labores de mantenimiento del equipo en la institución. La directora aceptó la propuesta y así es como él se encargó de instalar el equipo, brindando una inducción general a las docentes, en una iniciativa de gran valor para el proyecto.

En conjunto: dirección, docentes, padres y madres de familia, niños, niñas y Patronato Escolar programaron actividades para recaudar el dinero a través de eventos como miniferias, ventas y rifas. Meses después, el producto de todo este trabajo permitió adquirir cinco computadoras que fueron colocadas en cada aula, cuyo uso sería para los diez grupos de los ciclos de materno infantil y transición, resultando beneficiados aproximadamente 300 niños y niñas. El reto siguiente lo constituyó la capacitación en el uso de las tecnologías.

Mientras que algunas docentes demostraron facilidad en el manejo de tecnologías computacionales, otras tenían poco o ningún conocimiento al respecto. Sin embargo, gracias al apoyo mutuo, así como a la capacitación recibida por el padre de familia mencionado a través del TCU, el grupo de docentes logró salir adelante.

En el 2006, tanto el personal docente de la institución, como su directora, recibieron una capacitación en el uso de la computadora por parte del Programa Nacional de Informativa Educativa MEP-FOD. En ese mismo año, la directora de la Escuela Central de Tres Ríos, Ligia Cordero Pérez, planteó a la institución Preescolar la posibilidad de que los estudiantes recibieran informática en el laboratorio de la escuela, en tanto las profesoras de Informativa Educativa tuvieran lecciones disponibles que se pudieran orientar a este fin. La institución aceptó la propuesta y desde ese entonces todos los niños y niñas de Preescolar reciben dos lecciones semanales en el laboratorio de la escuela, ubicado a unos cuantos metros de sus respectivas aulas.

El trabajo en estos laboratorios se dificultó inicialmente, pues las estrategias pedagógicas de las lecciones estaban diseñadas para estudiantes de I Ciclo, además de que 
el manejo del comportamiento de los Preescolares se hacía difícil para las profesoras de cómputo. No obstante, luego del trabajo conjunto entre docentes de Preescolar e Informativa Educativa, la metodología mejoró paulatinamente y de forma sustancial. Este proceso de reestructuración de estrategias pedagógicas fue una ayuda muy importante para la labor de unas y otras docentes. En la actualidad, el apoyo se ha orientado hacia una inducción de los niños y niñas en el uso de la computadora y del soporte técnico básico. Para las docentes es claro que el complementar el uso del equipo en el aula, con estas lecciones semanales en el laboratorio, propicia que los niños manejen la máquina con mayor facilidad y en menor tiempo. Esto beneficia el manejo del equipo por sí mismos, una vez que están en el salón de clase. Desde entonces, y por iniciativa propia, algunas docentes motivadas para usar de una mejor manera la tecnología, han recibido cursos de capacitación, autofinanciados en la mayoría de los casos.

Para el año 2012, fueron 270 estudiantes, distribuidos en diez grupos, quienes tuvieron la posibilidad de accesar al equipo de cómputo instalado en sus salones de clase, con la ventaja de que se implementó el uso de la Internet en la institución y en cada una de las aulas, lo que ha incrementado aún más las posibilidades del uso para complementar las actividades diarias. Se realizan búsquedas sobre temas específicos, videos, canciones y cuentos animados, entre otros, los que les proporcionan material adicional que la docente considere conveniente para enriquecer los periodos de música y literatura entre otros.

\subsubsection{Metodologías implementadas y Software}

Las iniciativas de autogestión para obtener el equipo de cómputo en cada una de las instituciones mencionadas, se han desarrollado siguiendo dinámicas particulares, acordes con el contexto histórico y comunal de cada institución. En el Jardín José Ana Marín el proceso se inició con la incorporación de una computadora para uso exclusivo de las docentes, uso que se convirtió en necesario para ellas y que posteriormente se llevó al aula como un área más de trabajo; por su parte, en el Jardín Central de Tres Ríos, al implementar estrategias observadas en España e Israel, se incluyó el uso de la computadora dentro del salón de clase para uso de los niños y niñas. Si bien es cierto el proceso inició de forma diferente en ambas instituciones, la implementación final de metodología de trabajo dentro del aula fue parecida; así mismo, el tipo de software utilizado fue similar. Por tanto, en esta sección se tratan las experiencias de ambas instituciones de forma conjunta. 
La rutina diaria en las aulas Preescolares costarricenses está organizada en periodos, cada uno de ellos con un objetivo específico, dentro de los cuales se encuentra el periodo “Juego-Trabajo", donde los niños y niñas, de forma voluntaria, escogen diariamente el área a la que van a trabajar. Esas áreas están organizadas en diferentes espacios en el aula, cada una con materiales específicos, que promueven dinámicas de interacción y aprendizaje distintos; entre las que se encuentran el área de ciencias, la de construcción y la de arte, solo por nombrar algunas.

Según la información recolectada a partir de cuestionarios y las observaciones, ambas instituciones optaron por introducir el equipo de cómputo como un espacio nuevo, ofreciéndose dentro del aula como el área de "Cómputo". Las docentes de ambas instituciones mencionaron que dicha incorporación tuvo una excelente acogida por parte de los y las estudiantes. En las visitas realizadas a las aulas, se observó el interés por accesar el equipo, así como su deseo de escoger esa área en el periodo establecido. Por esa razón, es que para algunas maestras fue necesario el establecimiento de un control por semana que propiciara que todos y todas tuvieran oportunidad de usar la computadora. De lo contrario, muchos niños y niñas solicitaban utilizar el equipo varias veces, limitando el acceso a otros. De igual manera, las docentes promovieron que quienes poseían mayores conocimientos, apoyaran a aquellos que evidenciaron menor familiaridad con este tipo de tecnología, buscando así el fomento del trabajo colaborativo y la socialización

En las dos instituciones se procuró el mantenimiento de ciertas características del espacio físico, a saber: iluminación natural y ventilación, así como mobiliario adecuado y accesible para que uno o dos niños y niñas lo utilicen de manera confortable. En la mayoría de los casos, el equipo de cómputo del área cuenta con parlantes y dispositivos de $C D$ - $R O M$ que niños y niñas aprenden a manipular con facilidad y que son aprovechados para el mejoramiento de la experiencia interactiva con diferentes tipos de software educativos.

Las maestras reportaron que el software utilizado fue previamente analizado por ellas, de forma grupal en reuniones establecidas para tal fin, o individualmente y los resultados fueron compartidos con las compañeras de nivel. Las docentes pusieron en funcionamiento el juego y lo evaluaron, utilizando como criterios el vocabulario empleado, las imágenes, los colores, el sonido y los conceptos que se desarrollaban, que debían estar en correspondencia con los objetivos de los programas de estudio de los ciclos de transición o materno, así como con las edades e intereses de sus alumnos y alumnas. El software se 
aprobó o rechazó para su uso en el aula, dependiendo de si cumplía con los criterios antes mencionados.

El software (por ejemplo: "El conejo lector", "Aprendilandia" y "Trampolín") fue organizado según su dificultad, de manera que los niños reconocían los más sencillos y los que requerían mayores conocimientos, ellos mismos escogían los $C D-R O M$ de su preferencia. Las condiciones que generaron esta área de trabajo, permitieron que los niños y niñas regularan y desarrollaran diferentes dinámicas de acuerdo con la elección de los materiales.

Cada institución presentó otras particularidades, las docentes comentan que, por ejemplo, muchos de los estudiantes del Jardín de Niños José Ana Marín tienen acceso a la computadora en el hogar, por lo cual el proceso de introducción al trabajo en el aula se favoreció. Debido a esto, y en términos generales, algunas maestras realizaron un sondeo para determinar cuáles de sus estudiantes sabían usar la computadora. Una vez detectados, se les pidió que ayudaran a sus compañeros y compañeras. Otras docentes, en el periodo de conversación y durante una semana, explicaron las partes que conforman una computadora, así como generalidades sobre su uso y la utilización del software disponible.

En contraste, en el Jardín Central de Tres Ríos, los estudiantes tuvieron su primer contacto con la computadora hasta que se integraron a la institución y asistieron al Laboratorio de Cómputo de la Escuela, donde una docente de Informativa Educativa les explicó el funcionamiento y los cuidados que requiere el equipo, preparándolos para que fueran capaces de utilizarlo con mayor facilidad una vez en el aula.

Mediante la observación, se evidenció que durante las actividades en el área, los niños y niñas desarrollaron sus dinámicas de manera autónoma, donde la docente fue un apoyo ante eventuales dificultades. Así se intentó propiciar el desarrollo de seguridad y la noción de autoeficacia por parte de los estudiantes frente al equipo de cómputo.

\subsubsection{Alcances y limitaciones del proyecto}

La incorporación de la tecnología en las aulas de Preescolar mediante la autogestión, ha demostrado ser una estrategia viable para estas instituciones, proporcionando, además, otras ventajas que no habían sido contempladas inicialmente. El uso de la computadora se ha ampliado y ahora es visto como un recurso adicional para la docente, el equipo se utiliza en diferentes momentos, como en los periodos de conversación y experiencias de 
interacción, para iniciar o complementar los tópicos que se abordan mediante material interactivo.

Además, en algunos casos se ha introducido a la dinámica del aula la presentación de exposiciones por parte de los niños y niñas, utilizando programas para la proyección de diapositivas que han creado previamente en sus hogares con el apoyo de sus familias. Asimismo, el equipo se aprovecha en el periodo de literatura o música para la reproducción de canciones y cuentos. Comúnmente, cada actividad desarrollada en el aula, con el apoyo de la tecnología, queda documentada en el planeamiento didáctico en procura de orden y sistematización. Las reuniones de padres y madres, así como reuniones de personal, se han visto enriquecidas gracias a la incorporación de presentaciones digitales, videos educativos y/o formativos.

De acuerdo con el criterio de las docentes y tal como ha sido reportado (Vargas, 2003), niños y niñas tienen la oportunidad de aumentar sus niveles de atención y concentración por medio del uso de distintos programas, recursos que también se han llegado a convertir en un elemento más de motivación para asistir a la institución. Entre otras anotaciones, las docentes consideran que el espacio ofrece, además, materiales complementarios que pueden favorecer el ejercicio de habilidades de motora fina y coordinación psicomotriz, contribuyendo así a reforzar el trabajo que se realiza con otros materiales en diversos espacios del aula. También, señalan que el espacio cumple una importante función colaborando con el reforzamiento de conceptos de interés establecidos en los programas de estudio de Educación Preescolar. Entre otros, el software utilizado presenta actividades que demandan el manejo de conceptos como colores, números y formas.

Sin embargo, no todo el panorama es favorable. Estos proyectos, al haberse formulado justamente como iniciativas institucionales de autogestión, no reciben apoyo económico por parte del Ministerio de Educación, según lo comentan las directoras, lo cual dificulta la proyección de algunas mejoras como la adquisición de una segunda computadora por aula, lo que permitiría una mayor disponibilidad de equipo y, por ende, una mayor participación de los niños y niñas. Para algunas docentes, así como para Camacho (2009), proveer 2 computadoras por aula sería óptimo para el enriquecimiento tecnológico de los espacios Preescolares, siempre y cuando su incorporación se lleve a cabo de forma natural, propiciando que el equipo sea visto como otro recurso para el desarrollo en los diferentes ambientes de aprendizaje que existen. Del mismo modo, la ausencia de un mayor y 
constante apoyo económico limita el mantenimiento técnico y actualización de los equipos. Cuando sufre averías, la reparación tarda en realizarse por los escasos recursos para contratar el servicio, o bien, se debe esperar a que algún padre de familia u otra persona, con el conocimiento técnico requerido acceda a colaborar.

Para algunas docentes, otra limitación radica en su poca familiaridad inicial con los equipos de cómputo, por la carencia de alfabetización tecnológica específicamente dirigida hacia el contexto del aula Preescolar durante su formación profesional. Esto hace que el tiempo de planificación y búsqueda de nuevos materiales se dificulte un poco más en algunos casos, en comparación con lo que se percibe, de parte de otras colegas, que sí poseen mayor familiaridad con el equipo.

Dos últimas limitaciones percibidas por las maestras se vinculan con el acceso a la Internet y la necesidad de capacitación formal. Actualmente, una de las instituciones presentadas aquí, no cuenta con conexión a la Internet para los equipos que tienen en sus aulas, las maestras consideran que la adquisición de este recurso podría mejorar la productividad y eficiencia, así como aumentar las alternativas hacia nuevos usos. Para el segundo caso, y tomando en cuenta que las docentes se han visto en la necesidad de ir construyendo sus propias metodologías, basadas en su experiencia de aula y sin capacitación formal o el apoyo de material didáctico específico, se apunta la carencia de asesorías relacionadas con estos temas como otra limitación.

\section{CONCLUSIONES Y RECOMENDACIONES}

El carácter emergente de las iniciativas institucionales autogestionadas, para la adquisición de equipo de cómputo en algunos Preescolares públicos, evidencia un importante esfuerzo de por parte de grupos de docentes y administrativas por en buscar mejoras en las oportunidades de interacción y desarrollo que ofrece el aula de Preescolar a los niños y niñas. Al mismo tiempo, destaca la necesidad de atender la ausencia de lineamientos específicos dentro de las políticas públicas sobre el uso de las TIC en el sistema costarricense de Educación Preescolar, así como la buena disposición de muchas personas directa e indirectamente vinculadas con las comunidades de los jardines de niños, para participar y colaborar activamente con este tipo de proyectos.

Las estrategias de autogestión implementadas en estas dos instituciones, demostraron la capacidad que pueden tener los centros educativos Preescolares para lograr que sus 
alumnos tengan contacto con la tecnología. Lo anterior basado en una línea de acción conjunta, donde haya liderazgo y una visión compartida de lo que se desea obtener, involucrando a administrativos, docentes, padres y madres de familia, estudiantes y a la comunidad en general, esto por cuanto las personas valoran más lo que han obtenido mediante esfuerzo conjunto, asimilando la idea y logrando hacerla sostenible a través del tiempo.

Es importante tomar en cuenta que la sola presencia de las computadoras y la Internet en el aula no van a producir un aumento en la calidad de la educación; para ello, es necesario que docentes y administrativas incorporen estas herramientas novedosas, pero con un objetivo pedagógico, justificando la necesidad de su uso, ya sea como herramienta docente o como área de trabajo dentro del aula (Torres, 2010).

Resulta interesante que aunque las iniciativas comenzaron a ejecutarse de diferente forma en las dos instituciones descritas, ambas han establecido, finalmente, los equipos en sus dinámicas de aula como un área de trabajo más, lejos del concepto de Laboratorio de Cómputo, evitando así la escolarización, de modo tal que los niños y niñas pueden elegir voluntariamente el área de "Cómputo" una vez a la semana, área a la que están expuestos aproximadamente 45 minutos semanales, tiempo destinado al periodo "Juego-Trabajo".

Por su parte, la actividad de estos grupos de profesionales aporta evidencia importante que debe ser considerada por las autoridades respectivas, en su búsqueda de alternativas apropiadas para la incorporación de las TIC en la Educación Preescolar pública costarricense y en correspondencia con la visión de los programas de estudio.

Tanto el personal docente como administrativo manifiesta su satisfacción en tanto han percibido que iniciativas como estas realmente ayudan a reducir la llamada "brecha digital" entre sus comunidades de estudiantes. Sin embargo, también perciben claramente diferentes requerimientos que se hacen cada vez más necesarios para mejorar y darle sostenibilidad a estas iniciativas.

La capacitación formal en aspectos básicos de nivel técnico ayudaría, en gran medida, a que las docentes enfrenten de forma más eficiente algunos problemas que puedan presentar el equipo y los programas utilizados. Previamente, este aspecto ha sido considerado por Camacho (2009), cuando se apunta como una opción hacia el fortalecimiento del perfil de las docentes y, por tanto, nuevamente se sugiere aquí como una competencia de interés en la renovación de los perfiles de Educación Superior de docentes 
de Preescolar. No obstante, para aquellas que ya ejercen como maestras, programas de capacitación presencial y/o recursos en línea, podrían también convertirse en un apoyo valioso. Relacionado con esto, otro tipo de capacitación podría ser la impartida por especialistas, ya no centrada en el hardware únicamente, sino más bien en el software y en sus alternativas de uso como complemento para la consecución de objetivos propios de los currículos de Educación Preescolar, esta es otra necesidad que se percibe debe ser atendida.

La disponibilidad de software ha sido señalada como una debilidad que amerita ser considerada. Siendo el software un recurso central para aumentar y enriquecer las posibilidades que se les ofrece a los niños y niñas (Ferdig, 2006), preocupa el hecho de que el personal de las instituciones atraviese tantos problemas para adquirir programas educativos o juegos que respondan a la realidad del país y a los objetivos de los programas de estudios.

Importantes propuestas en el seno de instituciones, como la Fundación Omar Dengo (FOD), dentro del propio MEP, como el Programa Nacional de Innovaciones Educativas (PRONIE), o bien, dentro de la Universidad de Costa Rica, como en el caso del programa de Diseño y Desarrollo de Espacios Educativos con TIC o Centro de Investigaciones en Tecnología de la Información y Comunicación (CITIC) podrían ofrecer soporte a estas y otras necesidades de un importante sector de la educación costarricense.

Poco a poco, en nuestro país se han venido gestando esfuerzos importantes alrededor de temas de tecnología y educación y ahora se hace necesario que estos puedan articularse entre sí hacia el mejoramiento de la calidad de los sistemas educativos públicos. Iniciativas como el desarrollo de Ambientes Virtuales Colaborativos, por parte del CIN, y el LIIB de la Universidad de Costa Rica, en conjunto con el Departamento de Educación Preescolar del Ministerio de Educación Pública, han comenzado a dar algunos pasos en esta dirección, que deben ser también considerados.

En el corto plazo, la articulación de estos y muchos otros esfuerzos hacia el uso y desarrollo de software libre, que pueda ser ofrecido a docentes de todo el país sin costo y después de un riguroso diseño avalado por expertos, es una meta necesaria. Asimismo, el intercambio de experiencias entre docentes a nivel regional y nacional puede contarse como una de las estrategias exitosas del Ministerio de Educación Pública, que también podría favorecer el desarrollo de estos proyectos en el futuro próximo. 
Por último, llama la atención que aún no existan estudios, indicadores o evaluaciones claras que determinen el impacto del uso de esas tecnologías en las aulas de los diferentes niveles en el sistema educativo público nacional (Bujanda, Núñez, Quirós y Díaz, 2011). De manera similar, y al día de hoy, tampoco existen referencias específicas que confirmen algunos de los avances o progresos que las docentes perciben en dimensiones cognitivas y socio-afectivas de muchos de sus estudiantes por la utilización de las tecnologías. El estudio sistemático y el desarrollo de estrategias de seguimiento e investigación interdisciplinaria es fundamental para confirmar o descartar las percepciones referidas, o bien, para desarrollar un plan de cobertura y mejoramiento más eficiente respecto a las buenas y malas prácticas relativo al uso de las TIC en nuestros contextos educativos.

Todo lo anterior podría contribuir al mejoramiento del panorama para las instituciones que han desarrollado iniciativas autogestionadas, así como para sus comunidades vinculadas. Si bien docentes y administrativas se muestran satisfechas por los importantes logros y alcances de la experiencia, es también recurrente que hagan mención a la necesidad de un apoyo directo desde el Estado, el Ministerio de Educación y otras entidades, con el fin de dar sostenibilidad a los esfuerzos que ya se han hecho. En la misma dirección, parte de la satisfacción de estos grupos de profesionales parece estar relacionada con la verificación de su intuición inicial acerca del valor del equipo de cómputo como recurso dentro del aula.

Desde la experiencia en el aula Preescolar, niños y niñas se caracterizan por sus tendencias a la exploración autónoma de ambientes (Costa Rica, MEP, 2000), así como por sus iniciativas de comunicación abierta entre pares y hacia los adultos, lo cual se fomenta en el aula. En este contexto, recursos multimedia como los equipos de cómputo parecen corresponder adecuadamente con tales características. Estos recursos ofrecen todo un universo de oportunidades de conocimiento para los niños y niñas que por la edad en la que están son exploradores (Camacho y González, 2008), así como diversidad de variantes de estimulación interactiva por múltiples canales para aquellos estudiantes que se caracterizan por ser comunicativos. Claramente, el reto a futuro consistirá en aprovechar las ventajas asociadas con este tipo de recurso tecnológico en equilibrio con el resto de alternativas y recursos pedagógicos disponibles, valorando cada alternativa de forma crítica y desde la perspectiva integral de los programas de Educación Preescolar. 


\section{AGRADECIMIENTOS}

A la Ph.D. Ana Isabel Cerdas González, jefa del Departamento de Educación Preescolar del Ministerio de Educación Pública y a las Asesoras Nacionales de este departamento; a la M.Sc. Lorena Salas Soto, directora hasta el año 2010 del Jardín de Niños José Ana Marín así como a sus docentes; a la directora del Jardín Central de Tres Ríos, Licda. Flory Sanabria López y a las docentes de dicha institución. A todas ellas muchas gracias por su apoyo y colaboración en la realización de esta investigación.

\section{REFERENCIAS}

Bennett, Sue, Maton, Karl y Kervin, Lisa. (2008). The "Digital Natives" Debate: Critical Review of the Evidence. British Journal of Educational Technology, 39(5), 775-786.

Bujanda, María Eugenia; Núñez, Olmer; Quirós, Diego y Díaz, María Antonieta. (2011). Avance de los resultados sobre indicadores de tecnologías digitales en la educación primaria y secundaria de Costa Rica - Estudio de KERIS. Ponencia presentada en el II Congreso Internacional de Investigación Educativa 2011 en la Universidad de Costa Rica, San José, Costa Rica.

Cabra, Fabiola y Marciales, Gloria. (2009). Nativos Digitales: ¿Ocultamiento de Factores Generadores de Fracaso Escolar? Revista Iberoamericana de Educación, 50, 113-130.

Camacho, María. (2009). Desafíos de la educación inicial en la era digital (2008).Inter Sedes, 9(16), 69-88.

Camacho, María y González, Victoria. (2008). Principios para la Incorporación de Tecnología Digital en Espacios Preescolares. Inter Sedes, 9(17), 47-58.

Costa Rica, Ministerio de Educación Pública y Fundación Omar Dengo. (2011). Educación 360@.San José, Costa Rica: MEP-FOD.

Costa Rica, Ministerio de Educación Pública. (2000). Programa de Estudios Preescolar. San José, Costa Rica: MEP.

Costa Rica, Ministerio de Educación Pública. (2004). Concepción Curricular. San José, Costa Rica: Departamento de Educación Preescolar MEP.

Costa Rica, Departamento de Estadística Ministerio de Educación Pública. (2010). Instituciones Públicas que reciben Informativa Educativa. San José, Costa Rica.

Costa Rica. (2003). Reglamento General de Juntas de Educación y Juntas Administrativas № 31024-MEP.San José, Costa Rica: MEP. 
Federación Internacional Fe y Alegría (2009, octubre). Fe y Alegría: Expandiendo las oportunidades educativas de calidad en América Latina. Seminario Fe y Alegría Banco Mundial - Magis América, Lima, Perú.

Ferdig, Richard. (2006). Assessing technologies for teaching and learning: understanding the importance of technological pedagogical content knowledge. British Journal of Educational Technology 37(5), 749-760.

Garassini, María y Padrón, Clementina. (2004). Experiencias de uso de las TICs en la Educación Preescolar en Venezuela. Anales, 4(1), 221-239

Haugland, Susan y Wright, June. (2000). Young children and technology: $A$ world of discovery. New York: Allyn \& Bacon.

Haugland, Susan. (2000). Computers and Young Children. Recuperado de: http://ceep.crc.uiuc.edu/eecearchive/digests/2000/haugland00.pdf

Mesa, Fredy, Amador, Jenny, Sánchez, Claudia y Caro, Edgar. (2008). Unidad Didáctica para la Enseñanza de la Informática "Me divierto y Aprendo Pintando en Computador". Ponencia presentada en el IX Congreso Nacional de Informativa Educativa, Universidad del Norte, Barranquilla, Colombia.

México, Secretaría de Educación Pública (2011). Acuerdo número 592 por el que se establece la articulación de la Educación Básica. México, D. F.

Monge, Ricardo y Hewwitt, John. (2004). Tecnologías de la Información y las Comunicaciones (TICs) y el Futuro desarrollo de Costa Rica: El desafío de la exclusión. San José, Costa Rica: Fundación CAATEC.

Parra, Juan y Wodon, Quentin. (2011). Escuelas religiosas en América Latina: Estudios de Caso sobre Fe y Alegría. Washington, D.C. Banco Mundial.

Plowman, Lydia, Stephen, Christine, McPake, Joanna (2010). Supporting young children's learning with technology at home and in preschool. Research Papers in Education 25(1) 93-113.

Torres, María. (2010, setiembre). Tic y Educación. Apropiación de las Tecnologías de la Información y la Comunicación por Educadores Populares: Una experiencia de formación e intercambio docente en red. Ponencia presentada en el Congreso Iberoamericano en Educación, Buenos Aires, Argentina.

Vargas, Rodrigo. (2003). Escala de actitudes hacia la tecnología en el aprendizaje escolar aplicada a niños y niñas de primaria pública en Costa Rica. Análisis de validez y confiabilidad. Actualidades en Psicología, 19(106), 24-45.

Zúñiga, Magaly y Brenes, Melania. (2008). Estándares de desempeño de estudiantes en el aprendizaje con tecnologías digitales. Fundación Omar Dengo. Recuperado de http://www.fod.ac.cr/estandares/docs/estandares desempeno.pdf 